\title{
FOURIER TRANSFORMS AND MEASURE-PRESERVING TRANSFORMATIONS ${ }^{1}$
}

\section{O. CARRUTH McGEHEE}

\begin{abstract}
There exists a continuous function $f$ on the real line, vanishing at infinity, such that, for every measure-preserving transformation $h$, the composition $f \circ h$ fails to be a Fourier transform. This fact is a consequence of a theorem about measurable functions which is obtained from the theory of idempotents.
\end{abstract}

When $G$ is a locally compact abelian group, and $\Gamma$ is its dual group, let $A(G)$ denote the algebra of Fourier transforms of elements of $L^{1}(\Gamma)$, as described in Rudin's book [9, Chapter 1]. Let $Z, R$ and $T$ denote respectively the integer group, the real number system, and the circle group.

Jean-Pierre Kahane [4] adapted the work of P. J. Cohen and $H$. Davenport [3] to show that there is a function $f$ in $C_{0}(Z)$ such that for every permutation $p$ of the integers, $f \circ p$ fails to be in $A(Z)$. In this paper, the following result is obtained in a similar way.

THEOREM 1. There is a function $f$ in $C_{0}(R)$ such that for every measurepreserving transformation $h: R \rightarrow R, f \circ h$ fails to be in $A(R)$.

Theorem 1 is a consequence of the stronger Theorem 2 below, which concerns measurable functions, not just continuous ones. If $S$ is a Lebesguemeasurable set, let $|S|$ denote the measure of $S$. Let $L_{0}(R)$ denote the class of Lebesgue-measurable functions $f$ such that $|\{x:|f(x)|>\varepsilon\}|$ is finite for every $\varepsilon>0$.

THEOREM 2. For every positive number $s$, there exist small positive numbers $\alpha=\alpha(s)$ and $\varepsilon=\varepsilon(s)$ such that if $f \in L_{0}(R)$ and if

$$
|\{x: \varepsilon<|f(x)|<1\}|<\alpha|\{x:|f(x)| \geqq 1\}|,
$$

then there is a discrete measure $\mu \in M(R)$ such that $\|\hat{\mu}\|_{\infty} \leqq 1$ and $\left|\int f^{\prime} d \mu\right|>s$.

Theorem 2 implies that if $f \in L_{0}(R)$ and

$$
\left|\left\{x: \varepsilon(s)<s^{1 / 2}|f(x)|<1\right\}\right|<\alpha(s)\left|\left\{x: s^{1 / 2}|f(x)| \geqq 1\right\}\right|,
$$

Presented to the Society, April 27, 1973; received by the editors June 26, 1973.

AMS (MOS) subject classifications (1970). Primary 42A68; Secondary 43A25.

Key words and phrases. Fourier transforms, idempotents, measure-preserving transformations.

1 This work was supported in part by N.S.F. Grant GP-33583. 
then there is a discrete measure $\mu$ such that $\|\hat{\mu}\|_{\infty} \leqq 1$ and $\left|\int f d \mu\right|>\sqrt{ } s$. It is easy to construct a function $f \in C_{0}(R)$ such that (1) is satisfied for a sequence of values of $s$ tending to $\infty$. If $h$ is a measure-preserving transformation, then $f \circ h$ also satisfies (1) for the same values of $s$. If $M_{1}(R)$ denotes the space of discrete finite measures on $R$, then

$$
\sup \left\{\left|\int f \circ h d \mu\right|: \mu \in M_{1}(R),\|\hat{\mu}\|_{\infty} \leqq 1\right\}=\infty .
$$

Therefore $f \circ h$ cannot belong to $A(R)$, since

$$
\left|\int g d \mu\right| \leqq\|g\|_{A(R)}\|\hat{\mu}\|_{\infty} \text { for } g \in A(R), \quad \mu \in M(R) .
$$

Thus Theorem 1 follows from Theorem 2.

This work was done while trying to answer a question attributed to N. N. Lusin ([1, Volume 1, p. 330] or [2, p. 168]), which concerns homeomorphisms instead of measure-preserving transformations:

Is it true that for every continuous function $f$ on the circle group $T$ there is a homeomorphism $\varphi$ from $T$ onto $T$ such that $f \circ \varphi \in A(T)$ ?

Kahane's result, cited above, is that with $Z$ in the role of $T$, the answer is no. The answer for $T$ or $R$ is not known. For related work see [5] or [6, VII. 9], and [7] and [8].

We do not know how to prove a satisfactory analogue of Theorem 2 for the case of the circle group. We offer the following conjecture: For every $s>0$, there exist small positive numbers $\alpha=\alpha(s)$ and $\varepsilon=\varepsilon(s)$ such that if $f$ is a measurable function on $T$ and if

$$
|\{x: \varepsilon<|f(x)|<1\}|<\alpha \cdot \min \left\{|\{x:|f(x)| \geqq 1\}|,\left|f^{-1}(0)\right|\right\},
$$

then there is a discrete measure $\mu \in M(T)$ such that. $\|\hat{\mu}\|_{\infty} \leqq 1$ and $\left|\int f d \mu\right|>s$. It would follow from this result, of course, that there is a continuous function on $T$ of which no measure-preserving rearrangement is in $A(T)$.

It remains to prove Theorem 2. The next two results are from [3], and we omit the proof of the first one.

Lemma 1. Let $m_{1}, \cdots, m_{r}$ be integers, and let $z_{1}, \cdots, z_{r}$ be numbers of modulus 1 , where $r \geqq 3$. If $g$ is a trigonometric polynomial, $|g(x)| \leqq 1$ for all real $x$, and

$$
G(x)=g(x)\left\{1-2 r^{-2}-r^{-3} \sum_{i<j} \bar{z}_{i} z_{j} e\left(m_{i} x-m_{j} x\right)\right\}+r^{-5 / 2} \sum_{j} \bar{z}_{j} e\left(m_{j} x\right)
$$

(where $e(t)$ means $\left.e^{2 \pi i t}\right)$, then $|G(x)| \leqq 1$ for all real $x$. 
Lemma 2. Let $P, Q$ and $q$ be sets of integers, $Q \cap q=\varnothing, Q=\left\{n_{j}\right\}_{j=1}^{N}$, $n_{1}>n_{2}>\cdots>n_{N}$. For $p \in P$, let $N(p)$ be the number of integers in $Q \cup q$ that are greater than or equal to $p$. Let $r$ be an integer such that

$$
r+\frac{r(r-1)}{2} \sum_{p \in P} N(p)<N .
$$

Then there is a subset $\left\{m_{j}\right\}_{j=1}^{r}$ of $Q$ such that $m_{1}>m_{2}>\cdots>m_{r}$,

$$
\begin{aligned}
& p+m_{i}-m_{j} \notin Q \cup q \text { if } p \in P \text { and } i<j, \\
& m_{j}=n_{t(j)} \quad \text { where } t(j) \leqq j+\frac{j(j-1)}{2} \sum_{p \in P} N(p) .
\end{aligned}
$$

Proof. The $m_{j}$ 's may be chosen inductively. Let $m_{1}=n_{1}$. Having chosen $m_{j-1}$, let $m_{j}$ be the largest integer in $Q$ that is less than $m_{j-1}$ and satisfies (3). Condition (3) rules out at most $(j-1) \sum_{p \in P} N(p)$ integers, and therefore

$$
t(j)-t(j-1) \leqq 1+(j-1) \sum_{p \in P} N(p) .
$$

Statement (4) follows. Condition (2) assures that the process may be repeated $r$ times.

LEMMA 3. For every positive number $s$, there exist small positive numbers $a=a(s)$ and $\varepsilon=\varepsilon(s)$ such that, for all sufficiently large integers $N$, the following conditions hold. Let $Q$ and $q$ be disjoint sets of integers, $Q$ containing $N$ elements, $q$ containing no more than aN elements. Let $c$ be a function on $Z$ such that $|c(n)| \geqq 1$ for $n \in Q$ and $|c(n)|<\varepsilon$ for $n \notin Q \cup q$. Then there exists a trigonometric polynomial $g$ such that $\|g\|_{L^{\infty}(T)} \leqq 1$ and $\left|\sum_{n \in Z} c(n) \hat{g}(n)\right|>s$.

Proof. Let $r$ be an integer, $\sqrt{ } r>5 s$. Choose $a$ and $\varepsilon$ so that

$$
0<a<r^{-3 r^{2}-2}, \quad 0<\varepsilon<\sqrt{ } r /\left(20 \cdot 3^{r}\right) .
$$

It suffices to find a polynomial $g$ with these properties:

(i) $\|g\|_{L^{\infty}(T)} \leqq 1$,

(ii) $\hat{g}(n)=0$ for $n \in q$,

(iii) $\sum\{|\hat{g}(n)|: n \notin Q \cup q\}<3^{r}$,

(iv) $\sum_{n \in Q} c(n) \hat{g}(n)>\sqrt{ } r / 4$.

It follows from the last three conditions that

$$
\left|\sum_{n \in Z} c(n) \hat{g}(n)\right|>(\sqrt{ } r / 4)-\varepsilon 3^{r}>\sqrt{ } r / 5>s .
$$


Require $N>a^{-1}$. Let $Q$ be enumerated: $n_{1}>n_{2}>\cdots>n_{N}$. We shall construct a sequence of polynomials $g_{k}$, all satisfying conditions (i) and (ii), beginning with $g_{0}(x)=\left|c\left(n_{1}\right)\right| e\left(n_{1} x\right) \mid c\left(n_{1}\right)$. Finally, we shall let $g$ be $g_{k}$ for a suitable value of $k$ (namely, $k=r^{2}$ ). Suppose that $g_{k-1}$ has been defined. Let $P_{k-1}$ be the set of its frequencies:

If

$$
g_{k-1}(x)=\sum_{p \in P_{k-1}} \hat{g}_{k-1}(p) e(p x)
$$

$$
r+\frac{r(r-1)}{2} \sum_{p \in P_{k-1}} N(p)<N,
$$

then Lemma 2, with $P_{k-1}$ in the role of $P$, may be applied to obtain a set $\left\{m_{k i}\right\}_{i=1}^{r} \subset Q$. Let $z_{i}=c\left(m_{k i}\right) /\left|c\left(m_{k i}\right)\right|$ and let

$$
\begin{aligned}
g_{k}(x)= & g_{k-1}(x)\left\{1-2 r^{-2}-r^{-3} \sum_{i<j} \bar{z}_{i} z_{j} e\left(m_{k i} x-m_{k j} x\right)\right\} \\
& +r^{-5 / 2} \sum_{j} \bar{z}_{j} e\left(m_{k j} x\right) .
\end{aligned}
$$

By Lemma $1, g_{k}$ is bounded by one since $g_{k-1}$ is. The frequencies of $g_{k}$ are the integers in the set

and hence

$$
P_{k}=P_{k-1} \cup\left(P_{k-1}+\left\{m_{k i}-m_{k j}: i<j\right\}\right) \cup\left\{m_{k i}\right\},
$$

$$
\begin{aligned}
\sum_{p \in P_{k}} N(p) \leqq & \sum_{p \in P_{k-1}} N(p)+\sum_{i<j} \sum_{p \in P_{k-1}} N(p)+\sum_{j=1}^{r}(t(j)+a N) \\
\leqq & \left(1+\frac{r(r-1)}{2}\right) \sum_{p \in P_{k-1}} N(p) \\
& +\sum_{j}\left[j+\frac{j(j-1)}{2} \sum_{p \in P_{k-1}} N(p)+a N\right] \\
\leqq & \frac{r(r+1)}{2}+\left[1+\frac{r(r-1)}{2}+\frac{r(r+1)(2 r+1)}{12}-\frac{r(r+1)}{4}\right] \\
& \sum_{p \in P_{k-1}} N(p)+r a N \\
< & \left(r^{3} / 2\right) \sum_{p \in P_{k-1}} N(p)+r a N .
\end{aligned}
$$

Since $P_{0}=\left\{n_{1}\right\}$ and $N\left(n_{1}\right) \leqq a N$, by induction we obtain that $\sum_{p \in P_{k}} N(p)<$ $r^{3 k} a N$. By the restriction (5) on the choice of $a$, and for all $N>a^{-1},(6)$ is satisfied for $k \leqq r^{2}$. Let

$$
I_{k}=\sum_{n \in Q} c(n) \hat{g}_{k}(n) .
$$


Then $I_{0}=1$ and $I_{k} \geqq\left(1-2 r^{-2}\right) I_{k-1}+r^{-3 / 2}$. By induction,

$$
I_{k} \geqq(\sqrt{ } r / 2)-\left(1-2 r^{-2}\right)^{k}((\sqrt{ } r / 2)-1) .
$$

Therefore when $k=r^{2}, I_{k} \geqq(\sqrt{ } r / 2)\left(1-e^{-2}\right)>\sqrt{ } r / 4$, so that (iv) is established for $g=g_{k}$. For every $k$,

$$
\begin{aligned}
\sum_{n \in Z}\left|\hat{g}_{k}(n)\right| & \leqq \sum\left|\hat{g}_{k-1}(n)\right|\left(1-2 r^{-2}+r(r-1) / 2 r^{3}\right)+r^{-3 / 2} \\
& \leqq \sum\left|\hat{g}_{k-1}(n)\right|(1+1 / r) \\
& <(1+1 / r)^{k} .
\end{aligned}
$$

When $k=r^{2}$, this quantity is still less than $3^{r}$, and (iii) follows for $g=g_{k}$. Lemma 3 is proved.

Proof of Theorem 2. Given $s$, let $a$ and $\varepsilon$ be obtained as in Lemma 3 , and let $\alpha=a / 4$. Let $F=\{x: \varepsilon<|f(x)|<1\}, E=\{x:|f(x)| \geqq 1\}$, and suppose that $|F|<(a / 4)|E|$. We must show the existence of a suitable $\mu$.

Consider first the case when $|\{x \in E \cup F:|x|>b\}|=0$ for some finite $b$. Both the hypothesis, that $|F|<(a / 4)|E|$, and the desired conclusion are invariant under the change from $f(x)$ to $f(2 b x-b)$, and therefore we may suppose without loss of generality that $E \cup F \subseteq(0,1]$. Let $\eta>0$. There is a set $U \subset(0,1]$ which is the union of a finite number of open intervals, and such that the measure of the symmetric difference $E \nabla U$ is less than $\eta$. If $J$ is sufficiently large, then

$$
\left|\frac{1}{J} \sum_{j=0}^{J-1} \chi_{U}(x+j / J)-\right| U||<\eta \quad \text { for all } x \in[0,1 / J] .
$$

For arbitrary $J$,

$$
\int_{0}^{1} \chi_{E \nabla U}(x) d x=\int_{0}^{1 / J} \sum_{j=0}^{J-1} \chi_{E \nabla U}(x+j / J) d x=|E \nabla U|<\eta
$$

and

$$
\int_{0}^{1} \chi_{F}(x) d x=\int_{0}^{1 / J} \sum_{j=0}^{J-1} \chi_{F}(x+j / J) d x=|F|<(a / 4)|E| .
$$

Therefore there exists an $x$ such that both

$$
\sum_{j=0}^{J-1} \chi_{E \nabla U}(x+1 / j)<2 J \eta \text { and } \sum_{j=0}^{J-1} \chi_{F}(x+j / J)<J(a / 2)|E| .
$$

Therefore for every sufficiently large $J$, there is an $x$ such that

$$
\left|\frac{1}{J} \sum_{j=0}^{J-1} \chi_{E}(x+j \mid J)-\right| E||<3 \eta
$$


and

$$
\left|\frac{1}{J} \sum_{j=0}^{J-1} \chi_{F}(x+j / J)\right|<(a / 2)|E| .
$$

Let $Q=\{j: 0 \leqq j<J$ and $x+j \mid J \in E\}$. Then $Q$ has $N$ elements, where $N>J(|E|-3 \eta)$, so that by taking $J$ sufficiently large, we can make $N$ sufficiently large in the sense of Lemma 3 . By choosing $\eta$ sufficiently small and using (8) and (9), we may ensure that the set $q=\{j: 0 \leqq j<J$ and $x+j \mid J \in F\}$ has fewer than $a N$ elements. By Lemma 3, there is a polynomial $g(t)=\sum_{j} \hat{g}(j) e(j t)$ such that $\left|\sum_{j \in Z} \hat{g}(j) f(x+j \mid J)\right|>s$ and $|g(t)| \leqq 1$ for all $t$. Let $\mu$ be the measure that places mass $\hat{g}(j)$ at $x+j \mid J$. Then $\left|\int f d \mu\right|>s$ and $|\hat{\mu}(t)|=\left|\sum_{j} \hat{g}(j) e(-t(x+j \mid J))\right|=|g(-t \mid J)| \leqq 1$. Theorem 2 is proved in the case when $|(E \cup F) \backslash[-b, b]|=0$ for some $b$, and in particular for all $f$ with compact support.

Now to prove the theorem in the case of arbitrary $f$, let $E$ and $F$ be defined as before. Given $s$, let $\alpha$ and $\varepsilon$ be such that, whenever $|\{x: \varepsilon<|g(x)|<1\}|<\alpha|\{x:|g(x)| \geqq 1\}|$ and $g$ is measurable and has compact support, then there is a measure $v$ with finite support such that $\|\hat{v}\|_{\infty} \leqq 1$ and $\left|\int g d v\right|>3 s$. Suppose now that $|F|<\alpha|E|$. For $c>0$, let $V=V_{c}$ be the function in $A(R)$ defined so that $V(x)=1$ for $|x| \leqq c, V(x)=0$ for $|x| \geqq 2 c$, and $V(x)$ is linear on $[-2 c,-c]$ and on $[c, 2 c]$. Then $\|V\|_{A(R)}<3$. For $c$ sufficiently large,

$$
|\{x: \varepsilon<|V(x) f(x)|<1\}|<\alpha|\{x:|V(x) f(x)| \geqq 1\}|,
$$

and therefore there is a measure $v$ with finite support $Y$ such that $\|\hat{\nu}\|_{\infty} \leqq 1$ and $\left|\int V f d v\right|>3 s$. Let $A(Y)$ denote the algebra of restrictions to $Y$ of elements of $A(R)$, with norm

$$
\|g\|_{A(Y)}=\sup \left\{\left|\int g d \mu\right|: \mu \in M(Y),\|\hat{\mu}\|_{\infty} \leqq 1\right\} .
$$

Thus $\|V f\|_{A(Y)}>3 s$. But $\|V f\|_{A(Y)} \leqq 3\|f\|_{A(Y)}$. Hence $\|f\|_{A(Y)}>s$, so that there is a measure $\mu \in M(Y)$ such that $\|\mu\|_{\infty} \leqq 1$ and $\left|\int f d \mu\right|>s$.

Theorem 2 is proved.

\section{REFERENCES}

1. N. Bari, A treatise on trigonometric series. Vols. I, II, Macmillan, New York, 1964. MR 30 \#1347.

2. ——, Trigonometric series, Fizmatgiz, Moscow, 1961. MR 23 \#A3411.

3. H. Davenport, On a theorem of P. J. Cohen, Mathematika 7 (1960), 93-97. MR 23 \#A1992.

4. J.-P. Kahane, Sur les réarrangements des suites de coefficients de FourierLebesgue, C.R. Acad. Sci. Paris Sér. A-B 265 (1967), A310-A312. MR 37 \#4494.

5. - Sur les réarrangements de fonctions de la classe A, Studia Math. 31 (1968), 287-293. MR 39 \#6007. 
6. J.-P. Kahane, Séries de Fourier absolument convergentes, Ergebnisse der Math. und ihrer Grenzgebiete, Band 50, Springer-Verlag, Berlin and New York, 1970. MR 43 \#801.

7. - Brownian motion and harmonic analysis, Notes on lectures given at the University of Warwick in 1968.

8. O. C. McGehee, Helson sets in $T^{n}$, Lecture Notes in Math., no. 266, SpringerVerlag, Berlin and New York, 1971, pp. 229-237.

9. W. Rudin, Fourier analysis on groups, Interscience Tracts in Pure and Appl. Math., no. 12, Interscience, New York, 1962. MR 27 \#2808.

Department of Mathematics, Louisiana State University, Baton Rouge, LOUISIANA 70803 